\title{
La enseñanza en la Facultad de Farmacia y Bioquímica de la Universidad de Buenos Aires: tradiciones y perspectivas
}

Marilina Lipsman**

\begin{abstract}
The core question of our research has been how, since 1957, the teaching traditions of the School of Pharmacy and Biochemistry of the University of Buenos Aires (Facultad de Farmacia y Bioquímica de la Universidad de Buenos Aires-UBA) have been relating to the present practices. In order to understand the complexity of teaching, we had to get to know about the past of the teachers there involved, as well as about the traditions of institutional and disciplinary pedagogical practices in which teachers think and work. From a hermeneutic perspective we understood tradition and innovation as complementary processes. Within the frame of traditions we seek innovation, and in genuinely doing so we regain the best of traditions. Dialogue between traditions and present teaching has allowed us to recognize four perspectives that help us analyze the core question of our work: a) structural changes at the University; b) subjects giving rise to changes; c) the impact of changes within the institutional framework, and; d) the links between teaching and the disciplinary territory.
\end{abstract}

\section{RESUMEN}

Cómo se relacionan las tradiciones de enseñanza de la Facultad de Farmacia y Bioquímica de la UBA, con las prácticas presentes desde la perspectiva de la buena enseñanza desde 1957 constituyó la pregunta central de nuestro estudio de investigación. Entendimos que para comprender la enseñanza teníamos que sumergirnos en el pasado individual de los profesores y en las tradiciones de las prácticas pedagógicas institucionales y disciplinares donde los docentes piensan y trabajan. Desde una perspectiva hermenéutica comprendimos la tradición y la innovación como procesos complementarios. En el marco de las tradiciones buscamos la opción de innovar y en la innovación genuina recuperamos lo mejor de las tradiciones. A partir del diálogo entre tradición, actualidad y perspectiva en la enseñanza reconocimos cuatro perspectivas que permiten analizar el interrogante central del trabajo: a) los cambios estructurales de la Universidad, b) los sujetos que dan origen a los cambios, c) las repercusiones de los cambios en el marco institucional y d) los vínculos entre el territorio disciplinar y la enseñanza.

\section{NTRODUCCI ÓN}

En este proyecto de investigación abordamos ciertos aspectos de las problemáticas de la enseñanza de las Ciencias de la Salud en el nivel superior universitario e indagamos sobre las formas en que las tradiciones pasadas de enseñanza se entrelazan con las prácticas presentes desde la perspectiva de la buena enseñanza.

Para ello, nos propusimos identificar, describir y analizar las tradiciones en la enseñanza, originadas y vividas en el devenir de la Facultad de Farmacia y Bioquímica de la Universidad de Buenos Aires desde su creación en 1957 para vislumbrar qué permanece y qué cambia en el marco de las tradiciones en las propuestas vigentes; comprender e interpretar el sentido que asumen estas tradiciones para las prácticas de enseñanza de la Facultad en la actualidad y 
generar dimensiones de análisis que permitan comprender e interpretar las propuestas actuales que recuperen las tradiciones en el marco de la buena enseñanza (Fenstermacher, 1989; Litwin, 1999).

En este marco nos preguntamos ¿qué es lo que permanece y qué es lo que cambia en el marco de las tradiciones en las buenas propuestas de enseñanza vigentes? ¿Cuáles son las relaciones que se establecen entre las prácticas de producción del conocimiento, las prácticas profesionales y las prácticas de enseñanza? ¿Qué se enseñaba y cómo se enseñaba en el origen de la Facultad? ¿Qué se enseña y cómo se enseña hoy en el marco de las tradiciones de la Facultad?

\section{Acerca de los orígenes de las preguntas de investigación}

La presente investigación surgió en el marco de las tareas de formación docente, asesoramiento pedagógico y, especialmente, tareas de investigación que realizamos en el Área Pedagógica de la Facultad de Farmacia y Bioquímica de la Universidad de Buenos Aires.

En primer lugar, comenzamos esta investigación a partir de la reformulación -en un proceso dialéctico- de los resultados obtenidos en la investigación sobre las prácticas de enseñanza innovadoras en la facultad ${ }^{1}$ que realizamos durante los años 2001 y 2004 . En este proyecto buscamos identificar, analizar y comprender las prácticas de enseñanza innovadoras desarrolladas por los docentes de la Facultad.

Allí identificamos que las innovaciones no ocurren con independencia de las prácticas usuales y habituales que se vislumbran a priori como más tradicionales. Las innovaciones aparecen en un relato donde se marca -y a veces opone- "lo nuevo" y "lo viejo", se relaciona aquello que cambia y aquello que permanece, se entrelaza la tradición, la actualidad y la proyección. Así fue como situamos el foco de la investigación en la contracara de la innovación como espacio de indagación: las tradiciones de la enseñanza y sus perspectivas en la actualidad.

Diversos estudios muestran la relación entre lo nuevo y lo viejo, entre aquello que cambia y aquello que permanece. La originalidad pareciera actuar dentro del marco de la tradición, siendo una tradición totalmente nueva "uno de los sucesos más improbables" (Waring en Goodson; 2003).

De esta manera reconocimos lo particular, lo original de las buenas propuestas de clase, que permitieran rastrear aquellas primeras tradiciones que despertaron la preocupación por enseñar aquello que vale la pena ser enseñado.

Nos preguntarnos por la historia de estas prácticas y nos encaminamos al rastreo de las tradiciones y legados en la expresión de las prácticas actuales, no sólo de la enseñanza sino también las relativas a los diversos ámbitos en los que dichas tradiciones toman cuerpo (como las prácticas de la enseñanza, los espacios curriculares (teóricos, trabajos prácticos, residencias, entre otros), los planes de estudio, los espacios físicos de desarrollo de las prácticas (aulas y laboratorios), entre otros.

En esta búsqueda nos ocupamos de analizar cuáles eran las relaciones entre la enseñanza y la investigación dado que reconocíamos que las cátedras en la Facultad tienen un alto nivel de producción científica y esto debía, de alguna manera, estar presente en las prácticas pedagógicas. Indagamos el sentido que adquieren las prácticas de enseñanza en el contexto de las prácticas de producción de conocimientos, de investigación desarrolladas en la Facultad. Por ende, intentamos recuperar este sentido en las prácticas presentes, pero también en las pasadas, 
pues cada comunidad elabora en su contexto significados propios que construyen su identidad (Lemke, 1997). Así iniciamos nuestra búsqueda en la perspectiva de la buena enseñanza, dado que nos centramos en aquellas prácticas con fuerza moral y epistemológica, que comprometan a sus docentes en la tarea compleja y gratificante de educar a otros seres humanos (Fenstermacher, 1989).

Siguiendo estos supuestos, los objetivos de la investigación se definieron de la siguiente manera:

- Identificar, describir y analizar las tradiciones en la enseñanza, originadas y vividas en el devenir de la Facultad de Farmacia y Bioquímica de la Universidad de Buenos Aires desde su creación en 1957.

- Reconocer las continuidades y los cambios en el marco de las tradiciones en las propuestas de enseñanza vigentes.

- Comprender e interpretar el sentido que asumen estas tradiciones para las prácticas de enseñanza de la Facultad en la actualidad.

- Generar dimensiones de análisis que permitan comprender e interpretar las propuestas actuales que recuperen las tradiciones en el marco de la buena enseñanza.

\section{Consideraciones metodológicas}

Encuadramos este proyecto dentro de un enfoque cualitativo y crítico-interpretativo, por esto la comprensión del problema de estudio se realizó en colaboración con los actores cuyas prácticas se analizaron, se validaron las sucesivas construcciones teóricas con ellos y con especialistas del campo educativo y de las ciencias de la salud.

Adoptamos un punto de vista interpretativo, pues el significado primordial de este enfoque en la investigación sobre la enseñanza se refiere “...a cuestiones de contenido, más que de procedimiento. El interés en el contenido interpretativo lleva al investigador a buscar métodos que resulten apropiados para el estudio de ese contenido" (Ericsson: 1989: 197). Los estudios cualitativos parten de un conjunto menos específico de conceptos y sistemas clasificatorios vinculados a la teoría sustantiva que los elabora y reelabora en el curso de la investigación (Sautu, 2000).

Además, situamos esta investigación en una perspectiva narrativa. La narrativa oral -como la biografía o simplemente el relato cotidiano acerca de uno mismo- sería entonces una presentación más fiel de las experiencias personales que otros modos de expresión. Las historias relatadas permiten apreciar la manera en que los actores piensan la actividad docente y en general, las prácticas pedagógicas. Es necesario trascender los estados psicológicos individuales del docente para preguntarnos cómo surgieron tales estados mentales. "Para entender la docencia, tenemos que sumergirnos en el pasado, y no sólo en el pasado de los docentes individuales sino también en las tradiciones de práctica pedagógica dentro de cuya órbita los maestros piensan y trabajan" (McEwan, 1998).

La hermenéutica, la interpretación de los textos o documentos, de narraciones, de observaciones, nos permitieron encontrar sentidos y construir dimensiones para comprender ciertos aspectos del desarrollo de las prácticas, los acontecimientos, los actores involucrados y ocupados en la enseñaza desde la creación de la facultad hasta la actualidad. 
Desde esta perspectiva elaboramos acciones vinculadas a recabar y analizar información de diferentes actores y documentación que dieron cuenta de una mirada histórica de la enseñanza y así poder construir el primer sistema de categorías. Tomamos como fuentes de consulta: documentos producidos por la Facultad: memorias, registros, planes de estudio, recomendaciones y circulares, decisiones del Consejo Directivo de la Facultad, con el fin de profundizar nuestra visión sobre el objeto; trabajos de congresos presentados por docentes de la Facultad; una selección intencional de docentes a observar, registramos por medio de observaciones "sistemáticas amplias" las clases de estos. Asimismo, entrevistamos a los docentes observados (las observaciones fueron no participantes); tomamos entrevistas en profundidad (con su respectivo registro de grabación de audio) a reconocidos docentes de la comunidad académica de la Facultad y escribimos un diario de investigación.

Con un análisis inicial validamos los primeros resultados con los actores de la comunidad académica con los que trabajamos. Más tarde, buscamos la reinterpretación de las primeras categorías desde el reconocimiento de nuevas dimensiones de análisis, es decir, comenzamos la construcción de un segundo sistema de categorías. Con este segundo sistema, de doble hermenéutica, buscamos validar los resultados con expertos en el campo de la Didáctica del nivel superior y de las ciencias de la salud.

\section{Avances del proyecto}

Al estudiar las tradiciones en la enseñanza en el marco de las prácticas educativas vigentes en la Facultad de Farmacia y Bioquímica de la UBA, entendemos por "tradición" una forma de vida, un modo relativamente coherente y unificado de pensar, sentir y actuar dentro de un dominio concreto, en este caso el de la enseñanza. Una tradición tiene una larga y respetable historia, que ha comenzado hace tiempo, siendo más que un argumento intelectual: suscita sentimientos de adhesión y lealtad hacia un determinado punto de vista, implicando además, un compromiso con una serie de prácticas conexas. Plantear el problema en términos de "tradiciones" nos recuerda que ellas tienen una dimensión vivida que las convierte en mucho más que un argumento polémico (Jackson, 2002).

En este proceso hemos reconocido cuatro perspectivas de análisis a partir del diálogo entre la tradición, la innovación o cambio, la actualidad y las perspectivas de la enseñanza. Éstas son:

1. Los cambios estructurales de la Universidad y su vinculación con los cambios en la enseñanza: la universidad de elite y la universidad de masas

2. Los sujetos que dan origen a los cambios: individuos o grupos en las propuestas de enseñanza. Quiénes son los actores protagonistas de los cambios

3. Los cambios en los bordes o en el centro del currículo

4. Los vínculos entre el territorio disciplinar de la Farmacia y la Bioquímica y la enseñanza

\section{Los cambios estructurales de la Universidad y su vinculación con los cambios en la enseñanza: la universidad de elite y la universidad de masas}

Nuestro primer abordaje del objeto nos llevó a reconocer las tradiciones a partir del cambio profundo que ha sufrido la Universidad en estos últimos cincuenta o sesenta años, esto es, el pasaje de una institución de elite a otra con ciertas características de una organización de masas. Hemos encontrado ciertos datos que nos han permitido hipotetizar que algunas conformaciones de la buena enseñanza en la universidad de elite pueden perder su cualidad de significación y potencial de enseñanza en la universidad de masas, en especial, en aquellos momentos en que, por decisiones políticas, la apertura de la matrícula ha sido abrupta desde la perspectiva de los entrevistados (años 1974 y 1984). 
La cantidad de alumnos, para los docentes, fue un problema al que debieron dar respuesta formando criterios personales. Algunos optaron por organizar su cátedra multiplicando las ofertas que ya funcionaban, ampliaron la oferta de horarios teóricos y prácticos de forma tal de mantener la forma de enseñanza que creían valiosa y crearon nuevas espacios de enseñanza y formación:

"Los sábados de 18 a 22 hs realizaba una clase de repaso, síntesis o resumen de los temas tratados en la semana. A esta clase los alumnos iban por decisión propia" (Entrevista 1)².

Otros tomaron otras formas de trabajo: "En la época que tenía 35 no dábamos clases, las clases las daban los alumnos porque venían a discutir las cosas".

...en el 84/85 tuvimos 1200, y aprendimos otra cosa e hicimos otro modelo de enseñanza y dentro de los grupos elegíamos a aquellas personas que tenían mayor interés y con ellos trabajábamos como para mostrarle el laboratorio. ... donde cabían 40 personas de las $1200 . .$. que era la falsificación ideológica..." (Entrevista 2).

La masividad parece enfrentar a los docentes a dilemas éticos, las estructuras organizativas parecen no dar indicios para responder o generar buenas resoluciones para la enseñanza en esas condiciones. Aparecen elecciones o selecciones de grupos que pueden acceder a ciertos tesoros de conocimientos que se ven encarnados en los laboratorios, en los prácticos individuales, en las discusiones sobre el conocimiento con los maestros. De todas maneras parece que el ámbito que más se perjudicó fue el laboratorio: "Como alumno...vos tenías tu muestra y tenías que informar al final qué catión tenías y si no embocabas el catión te desaprobaban el trabajo práctico por más que tuvieras el escrito previo... así quedaban mesas vacías. Ahora se hace la mostración en los trabajos prácticos. La cuestión es cuando vos trabajás individualmente y tenés tu pipeta y tenés que responder por un resultado, cuando son dos la responsabilidad es compartida, no es lo mismo" (Entrevista 1).

Otra arista que cambió fue la relación entre profesores y alumnos en calidad de intercambios y encuentros. Con esto no afirmamos que la masividad haya sido la única variable en juego de este cambio.

“La relación del Profesor con los alumnos se perdió, su función...creo que el profesor tiene que dar una guía que conduzca, tiene que estar con el alumno, tiene que tener contacto con el alumno y eso no ocurre, eso está en manos de JTP" (Entrevista 3).

Así se marcan puntos nodales de conflicto o tensión: el acceso a las clases teóricas, el trabajo individual de laboratorio y el contacto con los profesores.

\section{Los sujetos que dan origen a los cambios: individuos o grupos en las propuestas de enseñanza. Quiénes son los actores protagonistas de los cambios}

A partir de las entrevistas realizadas reconocimos por lo menos dos tipos de cambios: los que surgen para resolver los problemas de la enseñanza y atenuar las dificultades planteadas por el devenir de las modificaciones que ha sufrido la Universidad (planteados en el punto anterior) y los que intentan romper con formas existentes aportando una mirada y justificación más amplia que la primera.

Sostenemos que "la innovación implica introducción o incorporación de algo nuevo a una realidad preexistente [donde] aparecen (...) aspectos innovadores de las nuevas propuestas, donde no todo es innovador. Las propuestas pueden ser parcialmente innovadoras cuando se modifican aspectos, instancias o partes del sistema vigente; son parciales cuando no rompen en su totalidad con las tradiciones de sus cátedras, o con el mismo sistema de regulación (...) Son innovadoras con respecto a algo y ese 'algo' refiere a 'algo vigente', 'tradicional', 'clásico', 'viejo' o 'anterior'" 
(Lipsman, 2002) sin embargo, pueden tener diversas justificaciones y significaciones. Algunas propuestas responden solamente a problemas de comprensión y otras se presentan como impacto en una práctica que ha sido mejorada o se configura como rupturista, en contra de las dinámicas actuales y tradicionales del campo de la enseñanza de FFyB.

Esta reelaboración y distinción del cambio y la innovación nos compelen a profundizar el análisis de las propuestas ubicadas en el segundo sentido presentado, entendiendo como innovación a las propuestas de carácter rupturista.

A partir del análisis del trabajo de campo encontramos cambios en las propuestas de enseñanza presentados como individuales o grupales. Nos preguntamos entonces quiénes son los sujetos de estos cambios y cuáles son las diferencias percibidas entre ellos. Cuando el cambio o la diferencia son buscados por los sujetos a partir de reconocer en lo "ajeno" los rasgos innovadores de otros docentes se ubicó el potencial innovador a partir de la seguridad y basándose en la experticia disciplinar, éste es vivido como un hito personal, individual, como una toma de conciencia singular.

"[Innovás o cambiás cuando] tenés seguridad con respecto a la disciplina" (Entrevista 4).

"Uno tiene muy claro lo disciplinar, por qué aplica ese contenido a la enseñanza" (Entrevista 5).

"Uno tiene más armas, uno conoce" (Entrevista 6).

"Uno toma conciencia de que existen otras formas de enseñar" (Entrevista 7).

Aquí la innovación no parece ser un producto de la reflexión y de la acción colaborativa de grupos de docentes, sino un logro estrictamente personal, al que se arriba solo. Podríamos interpretar que, en este caso, se trata de una innovación del autor el cual, a partir de su experticia puede tener movilidad para generar cambios genuinos.

En contrapunto reconocimos las propuestas de innovación llevadas adelante por equipos docentes organizados en el marco de una cátedra o departamento, que refieren a cambios grupales con impacto en diferentes ámbitos de la práctica docente: reuniones grupales, investigaciónacción, cambios en las propuestas de enseñanza, cambios en las ofertas educativas de la cátedra, etc. Estos grupos generalmente están compuestos por Jefes de Trabajos Prácticos y Auxiliares Docentes.

\section{Los límites, alcances y repercusiones de los cambios en el marco institucional}

En la tercera línea encontramos como recurrencia el surgimiento de propuestas actuales de cambio sobre aspectos que parecen no estar saldados en la cursada regular. Se producen cambios como complemento de la propuesta pedagógica de la cátedra más que como cambios en el centro del currículo. La innovación parece desarrollarse en espacios complementarios: espacios que acompañan y completan la propuesta de clase, de ejercitación y práctica, de formación tanto de alumnos como de auxiliares docentes jóvenes, de comunicación vía correo electrónico, de discusión sobre publicaciones científicas, entre otros.

En un espacio central, algunos profesores observados señalan que "el origen" de los cambios en la cátedra fueron la preocupación y el deseo de los docentes de su cátedra de mejorar la enseñanza, y éstos se encuentran en el centro del currículo: los trabajos prácticos.

"La idea fue de los JTP. Los JTP dijeron ¿y si hacemos un trabajo práctico creativo tipo mini beca? Y que los tipos vengan acá y lo trabajen. Y yo les pregunté: ¿ustedes van a asumir la consecuencia de esto, la plata que va a salir, el esfuerzo de ustedes por montar todo eso? ...es el cuarto año en que se hace esto" (Entrevista 8). 
Estos cambios tienen "el espíritu" o parecen retomar lo mejor de las tradiciones o formas de enseñanza de los profesores reconocidos por la comunidad en los años 60:

"En Química dábamos TP con una característica particular, de laboratorio abierto, no era el trabajo práctico que empieza a las 2 de la tarde y termina a las 3. Le dábamos problemas prácticos,... teníamos como un especie de becario a los estudiantes y lo reconocen, muchos de ellos" (Entrevista 3).

En las dos citas marcamos el peso del "nosotros" en el sostén y organización de los trabajos prácticos.

Así entendemos que los límites, alcances y repercusiones de los cambios en el marco institucional quedan enmarcados en las cátedras y a veces en las comisiones, pero que tienen anclaje en las tradiciones o formas de ver el mundo de la formación profesional y científica. Comprendemos que "la independencia relativa de los distintos ámbitos permite introducir innovaciones y cambios en determinados espacios sin que esto implique una modificación del conjunto de la organización. La adaptación localizada implica, en estos términos, también una baja propensión al cambio global, al mismo tiempo que una alta capacidad de sobrevivencia." (Krotsh, 1994: 109).

\section{Los vínculos entre el territorio disciplinar de la Farmacia, la Bioquímica y la enseñanza}

Los cambios y las permanencias de la enseñanza en la Facultad creemos que están relacionados con los cambios y los límites de las disciplinas que se transmiten. Sostenemos, siguiendo las ideas de Becher (2001), que la enseñanza en el ámbito universitario está vinculada a los límites de las disciplinas que se transmiten, "las formas de organización de la vida profesional de los grupos particulares de académicos están íntimamente relacionadas con las tareas intelectuales que desempeñan (...). Las prácticas habituales de una disciplina se correlacionan estrechamente con las características de los dominios de investigación pertinentes" (Becher, 2001:16). Se establecen vínculos entre lo social y lo cognitivo, "entre las tribus académicas y los territorios que habitan" (Becher, 2001: 21). Si bien la investigación de Becher está centrada en el área de investigación y él mismo nota que no ha indagado en el campo de la enseñanza, creemos que podemos utilizar su hipótesis y afirmar que en la enseñanza se reeditan y se ponen en juego las tensiones propias del campo disciplinar, la lucha por la independencia de otras áreas y la búsqueda de una identidad propia.

Desde diversas fuentes empíricas pudimos notar que la bioquímica es una disciplina que en la Argentina delimitó sus fronteras de manera particular en términos profesionales y académicos. En "sólo dos países se dicta como carrera de grado (España, Argentina y algunos países de Latinoamérica)"3. En otros territorios es un campo que los químicos, los médicos o los biólogos obtienen con estudios de posgrado. La disciplina es definida por sus miembros como un área que estudia la organización y el funcionamiento de sistemas biológicos, tienen una mirada físicoquímica biológica sobre la vida.

"La bioquímica fue formando un cuerpo doctrinario que pronto logró una independencia legítima. Si bien se dan en ella los dictados de la química y de la física, se fue imponiendo el criterio de que los fenómenos biológicos necesitaban un asentamiento en una nueva disciplina que tuvo los ribetes de interdisciplina primero y que luego asegurara su propia identidad (Iovine E, 2000: 11). 
Los límites del área son y fueron objeto de lucha. Afirmamos esto a partir de diferentes documentos que dan cuenta de los debates acerca de la creación de la Facultad y las notas que dan cuenta del rechazo que tuvo durante cuatro años (de 1954 a 1957) la solicitud de independencia de la Escuela de Farmacia y Bioquímica de la Facultad de Ciencias Médicas y su conformación como Facultad. La oposición estaba concentrada en la comunidad académica de la Facultad de Ciencias Exactas y Naturales y la profesional de químicos. Estaban en juego las incumbencias profesionales, se enfrentaban miradas profesionalistas y científicas de la universidad y se discutían los límites de la disciplina y su independencia como tal.

En 1956, frente al renovado pedido de la creación de la Facultad, la Asociación Química Argentina públicamente se opuso a esta solicitud con las siguientes palabras: "no parece justificado crear una nueva facultad que se especialice en una de las ramas de la química pues la universidad debe ser reestructurada sobre la base de Departamentos o Escuelas, antes que en Facultades en el sentido profesional que tienen en nuestro país" (Barrios Medina, 2006).

Luego de varios intentos, el lunes 13 de abril de 1957 se reabrió en el Consejo Superior la discusión sobre la creación de la Facultad de Farmacia y Bioquímica. Allí los límites de la disciplina volvieron a ser tema de debate. El representante de Ciencias Exactas y Naturales sostuvo: "tuve algunos puntos de vista que significan reparos a la creación, pero nada más que reparos, y no oposición". En ese sentido discrepó con la denominación propuesta de Facultad de Farmacia y Bioquímica y afirmó que daría su voto favorable a la creación de la Facultad si se llamara Farmacia, pues Bioquímica daría lugar a interferencias científicas y profesionales con la Facultad de Ciencias en la cual ya existía un doctorado en Bioquímica. El interventor de la Escuela de FyB replicó que esa interferencia no existía pues la bioquímica que "nosotros enseñamos" era la bioquímica humana u hospitalaria o clínica, y que la objeción era improcedente pues el título ya tenía treinta y siete años" (Barrios Medina, 2006).

Estos son debates por los límites de un campo, que tal como se presenta en las notas anteriores, configuraron el área de conocimientos con particularidades regionales (recordemos que en Europa y en países anglosajones, la Bioquímica es una especialización).

En esta discusión también se presenta la supuesta clara delimitación de Farmacia como una disciplina aplicada, con un campo profesional históricamente definido y arraigado a la tradición profesionalista universitaria argentina. La enseñanza de Farmacia se remonta a mediados del 1800: la Escuela de Farmacia fue creada específicamente en 1864. Así planteado parece no haber dudas sobre este campo como área profesional, pero la investigación comienza a tomar importancia a partir de 1920 y, por ende, los límites del campo también se amplían y se interrelacionan con los estudios bioquímicos y clínicos. Marca de esto son los trabajos sobre la historia de la disciplina de Barrios Medina y los documentos realizados por la Facultad en conmemoración de los 40 y 50 aniversarios de la facultad donde se enarbolan las áreas de investigación de Farmacia y de Bioquímica.

Alrededor de la creación de la facultad se generó una lucha por la definición de un campo propio y de una institución que lo cristalizara. Estas luchas no sólo se batieron en términos políticos, sino que se entramaron en las decisiones de macro y micro política de la enseñanza. En términos macro y como hito histórico encontramos el gran cambio de estructura curricular en 1959, y en el ámbito de la didáctica encontramos la preocupación por la especificidad bioquímica y farmacéutica de las ciencias básicas ubicadas como asignaturas en los primeros años de la carrera, la producción de libros específicos para resolver esta preocupación, y el diseño de formas de enseñanza que previeran la formación en la investigación "laboratorio abierto". 


\subsection{La definición disciplinar-profesional y los cambios curriculares}

La Facultad se creó a fines de la década del cincuenta en un contexto de desarrollo científico -técnico caracterizado por una serie de procesos institucionales que permitieron consolidar en la Argentina a la investigación como profesión. Por una parte se produce un importante movimiento de modernización de las universidades argentinas que se tradujo en una serie de innovaciones tales como la creación de la Universidad Nacional del Sur bajo el modelo departamental, el desarrollo de la autonomía en las universidades nacionales, el aumento significativo de las dedicaciones exclusivas docentes y particularmente en la Universidad de Buenos Aires bajo el rectorado de Risieri Frondizi, la creación del Consejo Nacional de Investigaciones Científicas y Tecnológicas (CONICET), del Instituto Nacional de Tecnología Agropecuaria (INTA) y el de Tecnología Industrial (INTI), entre otros.

Otra impronta de este impulso fue la consolidación de nuevas disciplinas en las Ciencias Sociales, tales como las carreras de Sociología y Psicología que mostraban un clima de renovación bajo las premisas del desarrollismo en materia económica y educativa. En este plano, la educación era parte del plan modernizador que iba a permitir pasar de una sociedad tradicional a una sociedad moderna, tal como lo postulaba el pensamiento de la CEPAL, que bajo el influjo de Raúl Prebisch, permitió dar a la investigación un importante impulso. No debería dejar de mencionarse que en este período se inicia un incremento constante y exponencial de la matrícula universitaria de alumnos universitarios: nuevas carreras, la feminización de la matrícula, el acceso de los sectores medios a la educación universitaria acompañado previamente por el desarrollo de las tasas de escolarización del nivel medio. Hacia 1958, por otra parte, se dicta la denominada Ley Domingorena, que permitió la creación de universidades privadas en el sistema educativo argentino.

En lo que se llamó la "Universidad de oro", la Facultad de Farmacia y Bioquímica se constituyó como tal, separándose de la Facultad de Ciencias Médicas y enfrentándose a la de Exactas. En ese contexto se brinda un gran apoyo a las investigaciones bioquímicas, y se crean institutos de Conicet.

En 1959 se realizó el cambio de plan de estudios más grande de la institución donde la carrera de Bioquímica dejó de ser una licenciatura que se obtenía luego de obtener el título de Farmacéutico. Se creó un ciclo común y los ciclos superiores de cada carrera. En el ciclo común se brindó mayor importancia a las llamadas ciencias básicas para brindar "fuertes fundamentos" y se organizaron las materias por cuatrimestre.

“...soy de la segunda camada del régimen cuatrimestral que se inicia en el 59, a los tres años de la creación de la facultad. Antes la carrera se regía por un régimen anual con los cuatro primeros años de Farmacia y los años posteriores para Bioquímica. Es decir, que las carreras no estaban bifurcadas, si vos primero hacías cuatro años de Farmacia, te recibías de Farmacéutico, hacías dos años más y te recibías de Bioquímico.

- ¿Sabe por qué se dio este cambio?

- Yo pienso que a partir de una preponderancia de la enseñanza de las ciencias básicas porque la idea del plan de estudios del régimen cuatrimestral era tener un ciclo básico, lo que hay es ciclo común, fuerte en materias básicas. Pero, el hecho de tener tres cursos de física, dos cursos de matemática, ya te da la pauta de que había una fuerte preponderancia de materias más bien de las ciencia duras. A partir de este régimen cuatrimestral la carrera se estructura sobre un Ciclo Básico Común a las dos carreras a partir del cual se abren los dos ciclos especializados, el de Farmacia y el de Bioquímica" (Entrevista 1). 
Los fundamentos de estos cambios estaban ligados a la necesidad de desarrollar profesionales con "buenos fundamentos" para la realización de estudios científicos en las dos áreas en que formaba la facultad. Ser referente científico pasó a ser uno de los mandatos del pasaje de escuela a facultad, impronta que se destaca en los documentos para conmemorar los 40 y 50 años de la facultad. Dichos escritos ponen el acento en el desarrollo de la investigación por medio de la creación de institutos de investigación y de cargos con dedicación exclusiva.

"El Decano Lugones y ese primer Consejo Directivo produjeron trascendentes cambios en la vida académica de la nueva facultad al implantar el régimen cuatrimestral de enseñanza de las materias a partir de 1959, reformulando a su vez el plan de estudios, designando nuevos profesores y favoreciendo la adopción de la dedicación exclusiva por un número importante de profesores y docentes auxiliares. La modalidad resultante del nuevo estilo de vida académica era similar a la de la Facultad de Ciencias Exactas, y favorecería grandemente la investigación científica asociada con el desarrollo académico"'.

La investigación parece erigirse como eje vertebrador de la nueva facultad, del cambio. Es objetivo, es meta. En las memorias las referencias a la enseñanza están en términos de cantidad de profesores y alumnos, pero no se presenta en modo de reflexión o problematización de la enseñanza, cuestión que sí encontramos en las historias de los entrevistados.

\subsection{Los límites y avances de la disciplina y la enseñanza en el aula 4.2.1. Las "básicas" para los bioquímicos y farmacéuticos}

Sostenemos que la lucha por los límites de las disciplinas atraviesan el campo de la enseñanza en la facultad desde su creación hasta estos días, especialmente en las materias que se dictan en los primeros años de la carrera que tienen como objeto de estudio a las ciencias básicas o puras-duras, según Becher, por ejemplo "matemática", "química inorgánica", "física" y que se enfrentan al problema de encontrar una particular configuración para dar cuenta de su especificidad en la formación de farmacéuticos y bioquímicos.

Esto lo afirmamos porque de diversas maneras los entrevistados dieron cuenta de la preocupación que se tiene en la institución sobre el recorte que se realiza de los contenidos, sus alcances y profundidad, la perspectiva epistémica y práctica que se toma en las materias del Ciclo Común. Por ejemplo un profesor emérito, reconocido por la comunidad académica, nos comenta que al asumir la titularidad de la asignatura de matemática en los años 50 reestructuró la materia:

"Lo primero que hice en matemática fue escribir un libro. Yo me dediqué a hacer esa matemática aplicada y la condensé en ese libro y ese libro tiene un poco más de lo que yo trabajaba en las clases pero no mucho más. ... la enseñanza de la asignatura a mi cargo tiene como base una ejercitación constante como ejemplos y problemas propios de su orientación científica y profesional" (Entrevista 3).

El problema era pensar la matemática para los farmacéuticos y bioquímicos. Para este entrevistado esta asignatura básica debe cobrar un carácter aplicado, de herramienta. El desafío era encontrar su utilidad y pertinencia, no descontextualizarla de los límites de las disciplinas que se impartían en la facultad.

"Sin olvidar los conocimientos básicos, en las páginas que siguen hemos mantenido siempre presente la finalidad de quien recibe la enseñanza. Estamos colaborando en la formación de un profesional diestro en el descubrimiento, síntesis y elaboración de las sustancias y productos 
que la medicina utiliza para mantener o devolver la salud al hombre. Este farmacéutico puede, además, orientar sus inquietudes hacia el estudio de la química de la materia viva cuyas alteraciones ayuda a corregir. El bioquímico resultante se halla en el dominio más apasionante de la biología: estudia el funcionamiento de la vida en sus detalles más íntimos" (Paladini, 1955: 7).

En la introducción a su libro de matemática Paladini subraya que los conocimientos básicos están en función de los perfiles profesionales de cada carrera, del estudio y cuidado de la salud y la vida desde una integración propia de la biología y la química.

Esta ocupación de encontrar lo propio a ser enseñado de las "básicas" en el Ciclo Común de las carreras es una tensión que hoy en día encontramos en los relatos de los docentes de la facultad. Los docentes de física hace aproximadamente diez años que discuten e innovan en la enseñanza de esta asignatura para promover el pensamiento, la lógica de investigación y los conocimientos que aporta la física para los farmacéuticos y bioquímicos (Diario de investigación).

Barnett afirma que "dada la complejidad interna de las distintas disciplinas y funciones que se relacionan entre sí y con la sociedad, se pueden contar diversas historias legítimas entre disciplinas y actividades académicas que están en tensión unas con otras" (Barnett, 2002: 51). Aquí la tensión aparece en lo que se dispone a ser enseñado en función del avance científico de las disciplinas de origen o básicas y lo distintivo y útil para las áreas profesionales y de conocimientos que se desarrollan en la Facultad.

\subsection{2. "Los trabajos prácticos como investigaciones acotadas"}

El impulso a la investigación es el elemento común que aparece cuando preguntamos a los entrevistados y a los docentes que observamos -que tuvieron o tienen a su cargo materias del Ciclo Superior- sobre los cambios que generaron en sus cátedras.

Diseñar el espacio de los trabajos prácticos como una investigación acotada o como un espacio abierto donde los tiempos de ejecución y análisis estén marcados por el experimento y no por los requerimientos organizativos de las normativas educativas, parece ser una innovación constante o una tradición que se renueva.

Podemos encontrar que en la cátedra de química biológica, en los años 60, los trabajos prácticos no tenían horario de salida; el Profesor los pensaba como "laboratorio abierto". "...en química biológica dábamos Trabajos Prácticos con una característica particular, de laboratorio abierto, no era el Trabajo Práctico que empieza a las 2 de la tarde y termina a las 3. Le dábamos problemas prácticos y el problema práctico no termina cuando termina el horario, termina cuando termina el experimento, entonces le dábamos el laboratorio abierto para que..., es decir teníamos como un especie de becario a los estudiantes y lo reconocen muchos, muchos de ellos. Uno de los alumnos que tuvimos en aquella época, que luego emigró a los EEUU, fue candidato serio al Premio Nobel y ahora lo acabamos de nombrar miembro correspondiente de la Academia Nacional de Medicina" (Entrevista 3).

En el año 2005 encontramos que la comunidad de la facultad presenta como innovación en la enseñanza que los alumnos elaboren sus propias determinaciones, que puedan diseñar el protocolo, realizarlo, recabar los datos, analizarlos e informarlos, hacer de la cursada un espacio donde el estudiante se proponga un desafío en términos de investigación en el área:

“- ¿Cuántos están cursando? 
- Unos 80. La mitad trabaja con una enfermedad no órgano-específica y la otra mitad con un modelo de diabetes y un anticuerpo, anti glutamato decarboxilasa. Los alumnos tienen que elaborar sus propios protocolos. Por supuesto que nosotros ponemos el ojo para que no elijan algo que no tenemos, instrumentos o algo, pero tienen libertad pautada.

... [Hay días específicos de prácticos que] son de actividad experimental, pero tal vez les cae un miércoles, un viernes, están incubando algo y tienen que venir en la mitad de la semana por más que no tengan clases".

"...Cuatro años atrás lo hacíamos por el método convencional, la guía de TP, hacíamos un simulacro, lo veían 80 tipos, dos o tres trabajaban, los demás miraban, todos pipeteaban, ningún acto creativo. Llegaba la autoevaluación de fin de año, que era anónima, nos ponían: los teóricos lindos", los trabajos prácticos -(el entrevistado hace una cara que comunica una valoración negativa por parte de los alumnos)- ya sabíamos. Los JTP dijeron ¿y si hacemos un trabajo práctico creativo?, ¿tipo mini beca? Y que los alumnos vengan acá y lo trabajen. Y yo les pregunté: ¿ustedes van a asumir la consecuencia de esto, la plata que va a salir, el esfuerzo de ustedes por montar todo eso? Bueno, ya este es el cuarto año en que se hace esto" (Entrevista 8).

Así aparecen las buenas prácticas de enseñanza en términos morales y epistemológicos, se presentan como valores: el conocimiento de la cocina de la investigación, del enfrentarse a retos de estudio, de "hacerse cargo" de los resultados y de las definiciones conceptuales y metodológicas que se toman. Es importante formar para ser investigadores y profesionales que pueden producir conocimiento y valor de enfrentarse al no saber, a los tiempos a veces indeterminados de los ensayos, a los errores, a los nuevos intentos, a los nuevos desafíos. Estas definiciones aparecen como improntas en el origen que por momentos parecen desintegrarse para volver a tener fuerza en las innovaciones. Son las tradiciones que se reeditan en forma creativa.

\section{PALABRAS FI NALES}

Creemos importante señalar que el par tradición-innovación no puede ser entendido como un par antinómico. Por el contrario, es en el marco de las tradiciones donde buscamos la opción de innovar. A su vez, es en la innovación genuina en donde queremos recuperar lo mejor de las tradiciones en el marco de la buena enseñanza, en la FFyB de la U.B.A.

Asimismo, entendemos que el estudio de la problemática educativa en cualquiera de sus aspectos, como el del cambio y permanencia en las tradiciones de la enseñanza, nos convoca siempre al reconocimiento de las articulaciones de ambos con la realidad política y social. En este sentido, y para ser coherentes con la adopción de un enfoque crítico-interpretativo, consideramos que el momento en el cual se ha ubicado este trabajo de investigación se vincula con cambios sustantivos en la universidad pública, transformaciones que involucran niveles políticos, pedagógicos, curriculares, financieros, entre otros, que siempre tenemos presentes dado que forman parte de la compleja realidad advertida en el recorte del objeto de investigación.

*Estos son los resultados del Proyecto de investigación UBACyT B100: La enseñanza en la Facultad de Farmacia y Bioquímica de la Universidad de Buenos Aires: tradiciones y perspectivas, dirigido por la Mg. Lipsman Marilina, en el cual han participado los miembros del Área Pedagógica de la FFyB: Augustovski, Ianina-Becaria; Florio, María Paz; Hara, Gabriela y Salvatierra, Fernando. 


\section{BI BLI OGRAFÍ A}

Barnett, (2002). Un cierto modo de conocimiento, en: Los límites de las competencias. El conocimiento, la educación superior y la sociedad. Buenos Aires, Argentina: Ed. Gedisa.

Becher, Tony (2001). Tribus y territorios académicos. La indagación intelectual y las culturas de las disciplinas. Barcelona, España: Ed. Gedisa.

Camilloni, A. y otras (1996). Corrientes didácticas contemporáneas. Buenos Aires: Ed. Paidós. Chaiklin, S. y Lave J. (comps) (2001). Estudiar las prácticas. Perspectivas sobre actividad y contexto. Buenos Aires : Amorrortu.

Erickson, F. (1989). Métodos cualitativos de investigación sobre la enseñanza, en Wittrock, M. (ed.) La Investigación de la enseñanza II. Métodos cualitativos y de observación. Buenos Aires : Ed. Paidós.

Fenstermacher, G. (1989). Tres aspectos de la filosofía de la investigación sobre la enseñanza, en: Wittrock, M. (comp.) La investigación de la enseñanza. Vol. I. Barcelona Ed. Paidós.

Fenstermacher, G. y Soltis, J. (1999). Enfoques de la enseñanza. Buenos Aires : Ed. Amorrortu. Fernández, L. (1994). Instituciones educativas. Buenos Aires : Ed. Paidós.

Gil Pérez, D. (1991). Qué hemos de saber y saber hacer los profesores de ciencias, Revista Enseñanza de las ciencias, vol. 9. Barcelona : Institut de Ciències de l'Educació de la Universitat Autònoma de Barcelona. Vicerectorat d'Investigació de la Universitat de València Goodson, I. (2003). Estudio del currículo. Buenos Aires, Argentina: Ed. Amorrortu.

Guijarro, R. B. y Messina Raimondi, G. (2000). Estado del arte sobre la innovaciones educativas en América Latina. Convenio Andrés Bello (Secab). Bogotá.

Hargreaves, A.; Earl, I.; Shawn, M.; Manning, S. (2001). Aprender a cambiar. Barcelona: Octaedro.

Huberman M. (1998). Trabajando con narrativas biográficas. En, La narrativa en la enseñanza, el aprendizaje y la investigación. Buenos Aires, Argentina: Ed. Amorrortu.

Jackson, P. (1999). Enseñanzas implícitas. Buenos Aires, Argentina: Ed. Amorrortu.

Jackson, P. (2002). Práctica de la enseñanza. Buenos Aires, Argentina: Ed. Amorrortu.

Kroscht, P. (1994). Organización, gobierno y evaluación universitaria, en: Kroscht, P. y Puiggrós A. Universidad y evaluación: estado del debate. Rei Argentina, Instituto de Estudios y Acción Social. Buenos Aires : Aique Editores.

Lemke, J. (1997). Aprender a hablar ciencia. España: Paidós.

Lipsman, M. (1997). Una práctica con informática, en Litwin, E. (coord.): Enseñanza e innovaciones en las aulas para el nuevo siglo. Buenos Aires, Argentina: Ed. El Ateneo.

Lipsman, M. (2002). Nuevas propuestas de evaluación en las prácticas de los docentes de la Facultad de Farmacia y Bioquímica de la Universidad de Buenos Aires. La innovación en la evaluación. Tesis de Maestría en Didáctica, Facultad de Filosofía y Letras, UBA.

Lipsman, M.; Fernández, B.; Schreier, L.; Wikinski, R. W. y otros (2002). "La capacitación docente en la Facultad de Farmacia y Bioquímica de la Universidad de Buenos Aires". Escuela de Graduados, Facultad de Farmacia y Bioquímica, UBA. Ponencia aprobada para ser presentada en la Sexta Junta Consultiva sobre el Postgrado en Iberoamérica de la Convención Universidad 2002. La Habana. 
Litwin, E. (1997). Las configuraciones didácticas. Una nueva agenda para la enseñanza superior. Buenos Aires : Ed. Paidós.

Litwin, E. (coord.) (1997). Enseñanza e innovaciones en las aulas para el nuevo siglo. Buenos Aires : Ed. El Ateneo.

McEwan, H. y Egan, K. (comps.) (1998). La narrativa en la enseñanza, el aprendizaje y la investigación. Buenos Aires :Ed. Amorrortu.

Meirieu, P. (2001). La opción de educar. Ética y pedagogía. Barcelona : Ed. Octaedro.

Popkewitz, T. (1994). Sociología política de las reformas educativas. Madrid : Ed. Morata.

Rivas Navarro, M. (2000). Innovación educativa. Teoría, procesos y estrategias. Madrid : Ed. Síntesis.

Sancho, J. y Hernández, F. (1993). La comprensión de la cultura de las innovaciones educativas como contrapunto a la homogeneización de la realidad escolar. Congreso Internacional de La Coruña. Ed. Mimeo.

Shulman, L. (1989). Paradigmas y programas de investigación en el estudio de la enseñanza: una perspectiva contemporánea, en: Wittrock, M. (comp.): La investigación de la enseñanza. Vol. I. Barcelona :Ed. Paidós.

Stenhouse, L. (1991). Investigación y desarrollo del currículo. Madrid : Ed. Morata.

Tishman, S.; Perkins, D.; y Jay, E. (1998). Un aula para pensar. Aprender y enseñar en una cultura de pensamiento. Buenos Aires: Ed. Aique.

Valles, M. (1999). Técnicas cualitativas de investigación social. España: Editorial Síntesis.

Wainerman, C. y Sautu, R. (2001). La trastienda de la investigación. Buenos Aires : Ed. Lumière.

\section{La historia en los documentos}

Paladini (1955). Matemáticas para estudiantes de Farmacia y Bioquímica. Editorial Harcor, Buenos Aires. Introducción.

Barrios Medina, Ariel (2006). Escuela de Farmacia y Bioquímica, Doctorado en Bioquímica y Farmacia, Facultad de Farmacia y Bioquímica. Un esbozo biográfico de cuatro generaciones. Trabajo premiado por la Academia Nacional de Farmacia y Bioquímica, 2006. Publicado en: http://www.ffyb.uba.ar/academia/Barrios\%20Medina.doc

Autobiografía inédita del Dr. Paladini (capítulos X -sin título- y IV: Palabras pronunciadas por Alcides R. Rega el 4 de abril de 1986 en el Acto Académico con motivo de la designación del Dr. Paladini como Profesor Emérito de la UBA).

Documento elaborado para el $40^{\circ}$ aniversario de la Facultad de Farmacia y Bioquímica. 19571997.

20 Años de Democracia en la Facultad de Farmacia y Bioquímica. Universidad de Buenos Aires. 2007.

Planes de Estudio de la Facultad

lovine Enrique (2000). Hitos Fundamentales en el desarrollo histórico de la Bioquímica. . Buenos Aires :Editoral Dunken. 
${ }^{1}$ Subsidio UBACyTB 013, "Enseñanza universitaria de las Ciencias Biomédicas: prácticas innovadoras en la Facultad de Farmacia y Bioquímica de la Universidad de Buenos Aires" dirigido por Marilina Lipsman

${ }^{2}$ Entrevistas realizadas a docentes de FFyB y a profesores eméritos de la comunidad académica de la institución.

${ }^{3}$ Diario de investigación, nota 2004.

${ }^{4} 50$ años Facultad de Farmacia y Bioquímica. pag.7

** Doctora (Cand.) en Educación, Universidad de Buenos Aires (UBA), Argentina. Especialista y Magíster en Didáctica, UBA. Licenciada en Ciencias de la Educación, UBA. Coordinadora de la Asesoría Pedagógica y Profesora Adjunta del Componente Pedagógico, Facultad de Farmacia y Bioquímica, UBA. Tutora, Master en Educación. 